\title{
Interferência do frênulo lingual nas funções do sistema sensorial motor oral em crianças: uma revisão sistemática
}

Interference of the lingual frenulum in the functions of the oral motor sensory system in children: a systematic review

Interferencia del frenillo lingual en las funciones del sistema sensorial motor oral en niños: una revisión sistemática

Recebido: 21/12/2020 | Revisado: 23/12/2020 | Aceito: 28/12/2020 | Publicado: 02/01/2021

Luisa Vargas Braz

ORCID: https://orcid.org/0000-0003-2965-8343 Universidade Federal de Santa Catarina, Brasil E-mail: luisavbraz@gmail.com

Laura Faustino Gonçalves

ORCID: https://orcid.org/0000-0002-0043-4349 Universidade Federal de Santa Catarina, Brasil E-mail:laurafaustinog@outlook.com

Patrícia Haas

ORCID: https://orcid.org/0000-0001-9797-7755

Universidade Federal de Santa Catarina, Brasil E-mail: patrícia.haas@ufsc.br

Ana Paula Blanco-Dutra

ORCID: https://orcid.org/0000-0002-6495-7480

Universidade Federal de Santa Catarina, Brasil E-mail: anapaulablancodutra@hotmail.com

\begin{abstract}
Resumo
Objetivo: Apresentar evidências científicas com base em revisão sistemática da literatura (PRISMA) sobre a interferência do frênulo lingual nas funções do sistema sensório motor oral em crianças. Metodologia: A busca de artigos foi realizada nas bases de dados Scielo, Lilacs, Pubmed, Scopus, Bireme e Web Of Science, não houve restrição de localização, período e idioma. Os descritores foram selecionados a partir do dicionário Descritores em Ciências da Saúde (DeCS) e Medical Subject Heading Terms (MeSH). Para complementar, foi realizada uma busca por literatura cinzenta no Google Scholar. Resultados: Inicialmente foram selecionados 131 artigos em todas as bases de buscas citadas, dimensionado para 121 após exclusão por repetição; em seguida, os títulos e abstracts foram analisados e 119 trabalhos foram excluídos pois não apresentavam aderência ao estudo proposto, portanto dois artigos foram admitidos para a análise final e total. Conclusão: As pesquisas que abordam a interferência do frênulo lingual nas funções orais, em sua maioria, se relacionam com a sucção e a amamentação, porém ainda são em pequeno número.
\end{abstract}

Palavras-chave: Freio lingual; Criança; Anquiloglossia.

\begin{abstract}
Objective: To present scientific evidence based on a systematic literature review (PRISMA) on the interference of the lingual frenulum in the functions of the oral motor sensory system in children. Methodology: The search for articles was carried out in the Scielo, Lilacs, Pubmed, Scopus, Bireme and Web Of Science databases, there was no restriction on location, period and language. The descriptors were selected from the Health Sciences Descriptors (DeCS) and Medical Subject Heading Terms (MeSH) dictionary. In addition, a search for gray literature was carried out on Google Scholar. Results: Initially, 131 articles were selected in all the cited search bases, scaled to 121 after exclusion by repetition; then, the titles and abstracts were analyzed and 119 papers were excluded because they did not adhere to the proposed study, therefore two articles were admitted for the final and total analysis. Conclusion: Research that addresses the interference of the lingual frenulum in oral functions, mostly, is related to sucking and breastfeeding, but they are still in small numbers.
\end{abstract}

Keywords: Lingual frenum; Child; Ankyloglossia.

\section{Resumen}

Objetivo: Presentar evidencia científica basada en una revisión sistemática de la literatura (PRISMA) sobre la interferencia del frenillo lingual en las funciones del sistema sensorio motor oral en niños. Metodología: La búsqueda de artículos se realizó en las bases de datos Scielo, Lilacs, Pubmed, Scopus, Bireme y Web Of Science, no hubo 
restricción de ubicación, época e idioma. Los descriptores se seleccionaron del diccionario Health Sciences Descriptors (DeCS) y Medical Subject Heading Terms (MeSH). Además, se realizó una búsqueda de literatura gris en Google Scholar. Resultados: Inicialmente se seleccionaron 131 artículos en todas las bases de búsqueda citadas, escaladas a 121 luego de exclusión por repetición; luego, se analizaron los títulos y resúmenes y se excluyeron 119 artículos por no adherirse al estudio propuesto, por lo que se admitieron dos artículos para el análisis final y total. Conclusión: Las investigaciones que abordan la interferencia del frenillo lingual en las funciones orales, en su mayoría, están relacionadas con la succión y la lactancia, pero aún son pequeñas.

Palabras clave: Frenillo lingual; Niños; Anquiloglosia.

\section{Introdução}

O frênulo lingual consiste em uma pequena prega de membrana mucosa ligada ao assoalho da língua. Esta membrana é formada por uma apoptose que, por volta da sétima semana de gestação ocorre na face ventral da língua, separando seus dois terços anteriores do assoalho da boca. Destaca-se que quando a apoptose não ocorre por completo durante o desenvolvimento embrionário, pode levar à anquiloglossia total ou parcial, também conhecida como "língua presa" e a qual pode delimitar os movimentos da língua (Martinelli, et al., 2015).

Segundo Brito et al. (2008) e Marchesan et al. (2012), a anquiloglossia é conhecida como uma anormalidade definida por alterações no frênulo lingual que resultam em movimento restrito da referida estrutura, podendo produzir dificuldades nas funções orais, como sucção, mastigação, deglutição e fala, as quais podem variar de acordo com o grau de alteração do frênulo. Os autores indicam que entre as alterações podem-se encontrar comprometimentos oclusais e periodontais resultando no rebaixamento da língua na cavidade oral (Tanigute, 2005).

Em relação ao impacto da alteração do frênulo lingual nas funções orofaciais, pode-se destacar a sucção, que está atrelada de modo direto com a amamentação e a deglutição (Martinelli; Marchesan; Berretin-Felix, 2013). Na sucção é observada uma simultaneidade de movimentos de várias estruturas. A alteração do frênulo lingual, como possível causa desta limitação do movimento, a língua não realiza os movimentos necessários para uma sucção efetiva, prejudicando a pega da mama e dificultando a retirada do leite, o que pode interferir na nutrição do bebê (Rios, 2005; Tanigute, 2005; Silva, et al., 2009; Martinelli, et al., 2013).

A sucção por sua vez, iniciada nos primeiros meses de vida, prepara a musculatura do bebê para a função mastigatória, a qual ocorre com mais eficácia a partir do $7^{\circ}$ mês de vida (Tanigute, 2005; Silva et al., 2009). A partir de um ano a um ano e meio a mastigação vai se tornar ainda mais efetiva com um padrão mais adulto, como consequência da estimulação de todo sistema estomatognático iniciada pela sucção. Esta estimulação evita o aparecimento de distúrbios miofuncionais, assim como distúrbios estruturais, dos ossos maxilares por exemplo, ao longo do crescimento (Tanigute, 2005; Bervian et al., 2008).

A mastigação também estimula os movimentos faciais adequados, sendo eles primordiais para a deglutição normal e consequentemente para a articulação precisa na fala da criança (Tanigute, 2005; Silva et al., 2009). A língua tem papel primordial para que a mastigação seja funcional, amassando o alimento contra o palato nos primeiros meses de vida e conduzindo o mesmo na cavidade oral, a partir da realização de movimentos de lateralização (Tanigute, 2005; Silva et al, 2009). Estudos afirmam que indivíduos com alteração no frênulo lingual possuem mais tendência a ter alterações na mastigação, além de alterações oclusais e periodontais, o que pode levar a adaptações durante a mastigação, deglutição e fala (Brito, et al., 2008; Silva, et al., 2009).

Com o rebaixamento da língua em pacientes com frênulo lingual alterado, estudos relatam observar alteração no espaço do palato duro, como também movimentos maxilares atípicos, o que pode gerar imprecisão articulatória (Gonçalves \& ferreiro, 2006; Brito, et al., 2008). Na fala, a alteração do frênulo lingual pode comprometer a execução de alguns fones, sendo os principais achados em [s] e [z], assim como a distorção de alguns sons alveolares como [t], [d], [n], [1] e [r] (Gonçalves \& Ferreiro, 2006; Brito et al., 2008). Estas distorções podem ocorrer devido ao fato de que os movimentos isolados da língua são 
imprecisos e movimentos articulatórios, como a elevação da ponta da língua, necessário para execução das consoantes alveolares, ficam prejudicados (Gonçalves \& Ferreiro, 2006).

\section{Objetivos}

A partir do exposto, a presente pesquisa apresenta como objetivo principal verificar a interferência do frênulo lingual nas funções do sistema sensorial motor oral em crianças (até dois anos de idade), visando responder a seguinte pergunta norteadora de pesquisa: Qual a interferência do frênulo lingual nas funções do sistema sensório motor oral em crianças?

\section{Métodos}

\subsection{Protocolo e registro}

A presente revisão sistemática foi conduzida conforme as recomendações PRISMA (Preferred Reporting Items for Systematic reviews and Meta-Analyses) (Moher et al., 2015). As buscas por artigos científicos foram conduzidas por dois pesquisadores independentes nas bases de dados eletrônicas MEDLINE (Pubmed), LILACS, SCIELO, SCOPUS, WEB OF SCIENCE e BIREME, sem restrição de idioma, período e localização. Para complementar, foi realizada uma busca por literatura cinzenta no Google Scholar.

A pesquisa foi estruturada e organizada na forma PICOS, que representa um acrônimo para População alvo, a Intervenção, Comparação e “Outcomes” (desfechos). População de interesse ou problema de saúde (P) corresponde a crianças (até dois anos de idade); intervenção (I): frênulo lingual; $(\mathrm{C})$, sistema sensório motor oral; outcome $(\mathrm{O})$ : interferência $(\mathrm{S})$ : estudo descritivo, estudo transversal, estudo observacional, relatos de caso, estudos de caso-controle, ensaios clínicos controlados, estudos de coorte.

Tabela 1. Descrição Dos Componentes Do Picos.

\begin{tabular}{l|l}
\hline \multicolumn{1}{c|}{ Acrônimo } & \multicolumn{1}{c}{ Definição } \\
\hline $\mathbf{P}$ & Crianças \\
\hline $\mathbf{I}$ & Frênulo Lingual \\
\hline $\mathbf{C}$ & Sistema sensório motor oral \\
\hline $\mathbf{O}$ & Interferência \\
\hline $\mathbf{S}$ & $\begin{array}{l}\text { Estudo descritivo } \\
\text { Estudo transversal } \\
\end{array}$ \\
Estudo observacional \\
Relatos de caso \\
Estudos de caso-controle \\
Ensaios clínicos controlados \\
Estudos de coorte
\end{tabular}

Fonte: Autores. 


\subsection{Estratégia de pesquisa}

Os descritores foram selecionados a partir do dicionário Descritores em Ciências da Saúde (DeCS) e Medical Subject Heading Terms (MeSH), haja vista a sua grande utilização pela comunidade científica para a indexação de artigos na base de dados PubMed. Diante da busca dos descritores, foi realizada a adequação para as outras bases utilizadas. Foram propostas para as buscas os seguintes descritores: (Lingual Frenum) (Stomatognathic System) (Children), e o operador booleano: and. A busca ocorreu em agosto de 2020.

\subsection{Critérios de Elegibilidade}

O desenho dos estudos selecionados foi do tipo transversal prospectivo e retrospectivo. Foram incluídos nesta pesquisa estudos sem restrição de idioma, período e localização. A Tabela 2 representa os critérios de inclusão e exclusão desenvolvidos nesta pesquisa. Os estudos obtiveram pontuação 11 no protocolo modificado de Pithon et al. (2015) para avaliação da qualidade dos mesmos. Foram inclusos estudos com crianças de até dois anos de idade.

\subsection{Critérios de inclusão}

A presente revisão incluiu estudos publicados sobre interferência do frênulo lingual nas funções do sistema sensorial motor oral em crianças (até dois anos de idade). Os desenhos dos estudos selecionados foram relatos de casos, estudos de casos e controle, ensaios clínicos controlados, estudos de coorte, estudos em triagem, estudos observacionais e estudos randomizados.

\subsection{Critérios de exclusão}

Foram excluídos estudos publicados no formato: Cartas ao editor, diretrizes, revisões sistemáticas, meta-análises e resumos. Estudos que não tenham descrito ou que foram pouco claros ou indisponíveis.

\subsection{Risco de viés}

A qualidade dos métodos utilizados no estudo incluído foi avaliada pelos revisores de forma independente ( $\mathrm{PH} \mathrm{e}$ APBD), de acordo com a recomendação PRISMA (Moher et al., 2015). A avaliação priorizou a descrição clara das informações. Neste ponto, a revisão foi realizada às cegas, mascarando os nomes dos autores e revistas, evitando qualquer viés potencial e conflito de interesses.

\subsection{Análise dos dados}

A extração dos dados para o processo de elegibilidade dos estudos foi realizada utilizando-se uma ficha própria para revisão sistemática elaborada por dois pesquisadores em Programa Excel ${ }^{\circledR}$, na qual os dados extraídos foram adicionados inicialmente por um dos pesquisadores e, então, conferidos por outro pesquisador. Inicialmente foram selecionados de acordo com o título; em seguida, os resumos foram analisados e apenas os que fossem potencialmente elegíveis foram selecionados para a próxima fase de avaliação. Com base nos resumos, artigos foram selecionados para leitura integral e foram admitidos os que atendiam a todos os critérios pré-determinados e respondiam a pergunta norteadora. Em caso de desacordo entre avaliadores, um terceiro avaliador (APBD) tomou a decisão sobre a elegibilidade do estudo em questão.

\subsection{Forma de seleção dos estudos}

Inicialmente os revisores de elegibilidade (PH e LFG) foram calibrados para a realização da revisão sistemática por APBD e LVB. Após a calibração e esclarecimentos de dúvidas, os títulos e resumos foram examinados por dois revisores de 
elegibilidade (PH e LFG), de forma independente, os quais não estavam cegos para o nome dos autores e das revistas. Aqueles que apresentaram um título dentro do âmbito, mas os resumos não estavam disponíveis, também foram obtidos e analisados na íntegra. Foram excluídos estudos fora do âmbito, relatos de caso, cartas ao editor e/ou editorial, revisões de literatura, índices, resumos e estudos em animais. Posteriormente, os estudos elegíveis preliminarmente tiveram o texto completo obtido e avaliado. Em casos específicos, quando o estudo com potencial de elegibilidade apresentasse dados incompletos, os autores foram contatados por e-mail para mais informações. Na inexistência de acordo entre os revisores, um terceiro (APBD) foi envolvido para a decisão final.

\subsection{Dados Coletados}

Após a triagem, o texto do artigo selecionado foi revisado e extraído de forma padronizada por dois autores ( $\mathrm{PH}$ e LFG) sob a supervisão de APBD e LVB, identificando-se ano de publicação, local da pesquisa, idioma de publicação, tipo de estudo, amostra, método, resultado e conclusão do estudo.

\subsection{Resultado clínico}

O resultado clínico de interesse foi analisar a interferência do frênulo lingual nas funções do sistema sensório motor oral em crianças. Aqueles que não utilizaram a abordagem sobre a interferência do frênulo lingual nas funções do sistema sensório motor oral em crianças não fizeram parte da amostra da revisão de literatura.

\section{Resultados}

A partir dos descritores eleitos, os bancos de dados foram consultados e foram obtidos os resultados disponibilizados na Tabela 2. 
Research, Society and Development, v. 10, n.1, e3510111396, 2021 (CC BY 4.0) | ISSN 2525-3409 | DOI: http://dx.doi.org/10.33448/rsd-v10i1.11396

Tabela 2. Classificação das referências obtidas nas bases de dados Pubmed, Scielo, Lilacs, Web Of Science e Scopus.

\begin{tabular}{|c|c|c|c|c|c|}
\hline Descritores & $\mathbf{N}^{\mathbf{o}}$ & $\begin{array}{c}\text { Referências } \\
\text { excluídas }\end{array}$ & Motivo & Selecionado & Banco de dados \\
\hline $\begin{array}{l}\text { (lingual } \\
\text { Frenum) and } \\
\text { (Stomatognathic } \\
\text { System) and } \\
\text { (children) }\end{array}$ & 120 & 118 & \begin{tabular}{|} 
Duplicados (8); \\
excluídos por \\
abstract (20); \\
xcluídos por título \\
$(90)$
\end{tabular} & 2 & Pubmed \\
\hline $\begin{array}{l}\text { (lingual Frenum) } \\
\text { and } \\
\text { (Stomatognathic } \\
\text { System) and } \\
\text { (children) }\end{array}$ & 5 & 5 & $\begin{array}{l}\text { Excluídos por } \\
\text { título (2); } \\
\text { excluídos por } \\
\text { abstract (2); } \\
\text { duplicado (1) }\end{array}$ & 0 & Lilacs \\
\hline $\begin{array}{l}\text { (lingual } \\
\text { Frenum) and } \\
\text { (Stomatognathic } \\
\text { System) and } \\
\text { (children) } \\
\end{array}$ & 0 & 0 & - & - & Scielo \\
\hline $\begin{array}{l}\text { (lingual } \\
\text { Frenum) and } \\
\text { (Stomatognathic } \\
\text { System) and } \\
\text { (children) } \\
\end{array}$ & 0 & 0 & - & - & $\begin{array}{l}\text { WEB OF } \\
\text { SCIENCE }\end{array}$ \\
\hline $\begin{array}{l}\text { (lingual } \\
\text { Frenum) and } \\
\text { (Stomatognathic } \\
\text { System) and } \\
\text { (children) } \\
\end{array}$ & 9 & 9 & $\begin{array}{c}\text { Duplicados (4); } \\
\text { xcluídos por título } \\
(5)\end{array}$ & - & Bireme \\
\hline $\begin{array}{l}\text { (lingual } \\
\text { Frenum) and } \\
\text { (Stomatognathic } \\
\text { System) and } \\
\text { (children) }\end{array}$ & 0 & 0 & - & - & SCOPUS \\
\hline Total & 134 & 132 & & 2 & Pubmed \\
\hline
\end{tabular}

Fonte: Autores.

Inicialmente foram selecionados 131 artigos em todas as bases de buscas citadas, dimensionado para 121 após exclusão por repetição; em seguida, os títulos e abstracts foram analisados e 119 trabalhos foram excluídos, pois não apresentavam aderência ao estudo proposto, portanto dois artigos foram admitidos para a análise final e total. Os estudos selecionados (Ngerncham, et al., 2013; Pransky, et al.,2015) para a pesquisa foram do tipo estudo transversal prospectivo (Ngerncham, et al.,2013) e retrospectivo (Pransky, et al., 2015) utilizados para o desenvolvimento desta pesquisa (Figura 1). 
Research, Society and Development, v. 10, n.1, e3510111396, 2021

(CC BY 4.0) | ISSN 2525-3409 | DOI: http://dx.doi.org/10.33448/rsd-v10i1.11396

Figura 1. Fluxograma de busca e análise dos artigos.

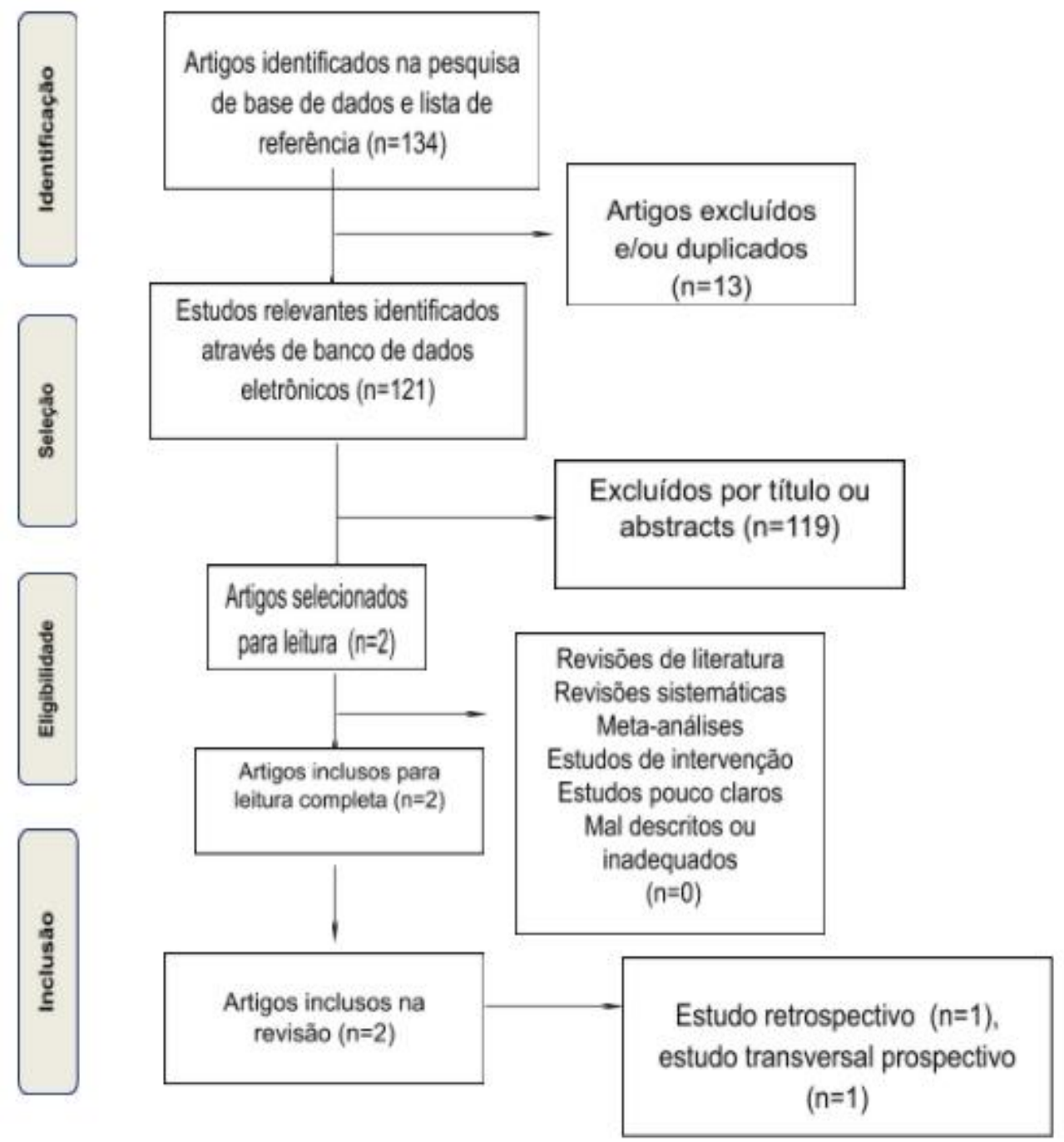

Fonte: Autores.

O primeiro estudo (Ngerncham, et al.,2013) objetivou determinar a prevalência de recém-nascidos com anquiloglossia e possíveis dificuldades subsequentes de amamentação, assim como outros fatores que afetam a eficácia da amamentação. Para o estudo foram recrutadas 2.679 mulheres no pós-parto e seus respectivos bebês. O exame físico dos bebês e das mães, assim como as práticas de amamentação foi avaliado entre 24 e 48 horas de vida dos recém-nascidos. O exame incluiu a aparência do frênulo lingual do bebê e a anatomia do mamilo (após a sucção) das mães. Contudo, durante a avaliação, as mães foram solicitadas a iniciar a amamentação e, após um curto intervalo, descrever o local no qual sentiam a língua de seu bebê. Utilizando apenas critérios anatômicos. A anquiloglossia destacada foi classificada como grave em $\mathrm{n}=428$ (16\%) lactentes e moderada em $n=600(22,4 \%)$. As proporções entre os sexos masculino e feminino para presença de alteração de frênulo lingual moderada e grave foram, respectivamente, 1,1:1, e 1,8:1 (Ngerncham, et al.,2013).

Como resultado (Ngerncham, et al.,2013) cerca de $n=247$ bebês $(9,2 \%)$ foram diagnosticados com dificuldades de amamentação. As taxas de dificuldade para amamentar bebês com anquiloglossia moderada e grave foram de 11,8\% (71/600 bebês) e 37,9\% (162/428 bebês), respectivamente. Ademais, fatores significativamente associados ao aumento do risco de dificuldades de amamentação foram anquiloglossia de classificação moderada e grave, mamilos curtos, mães que relataram a 
não sensação da língua do bebê, ou sentir a língua do bebê em seu mamilo, contudo destacaram ser mãe pela primeira vez. Apenas os bebês que ainda apresentavam dificuldade para amamentar associada à anquiloglossia foram aconselhados para tratamento conservador ou frenotomia (Ngerncham, et al.,2013).

O segundo estudo (Pransky, et al., 2015) foi composto por 618 bebês e suas mães. A população do estudo foi composta por bebês saudáveis sem outros problemas médicos significativos. Todos os bebês foram submetidos a um exame completo de cabeça e pescoço, que incluiu palpação do assoalho da boca e frênulo lingual. A anquiloglossia, se presente, foi então classificada como subtipos anterior (tipos I e II) ou posterior (tipos III e IV). As mães foram então questionadas sobre a amamentação do seu bebê e as dificuldades de amamentar, como era a pega do bebê durante a amamentação. Havia $\mathrm{n}=362$ (59\%) bebês do sexo masculino e $\mathrm{n}=256$ (41\%) do sexo feminino. Cerca de $\mathrm{n}=127$ (21\%) dos $\mathrm{n}=618$ não apresentaram anomalias da cavidade oral notadas no exame físico e, portanto, não tiveram intervenção. Das que apresentavam dificuldades para amamentar e anomalias da cavidade oral, a maioria $(n=290,47 \%)$ apresentava anquiloglossia anterior. Houve $(n=120$, $19 \%)$ lactentes que foram considerados como tendo anquiloglossia posterior e $(n=14,2 \%)$ foram diagnosticados com ligamento do lábio superior. Trinta e quatro $(6 \%)$ lactentes apresentavam anquiloglossia anterior e ligamento no lábio superior, enquanto ( $\mathrm{n}=33,5 \%)$ apresentavam anquiloglossia posterior e ligamento no lábio superior. Todos os bebês que apresentavam dificuldades para amamentar foram encaminhados para realização da cirurgia (Pransky, et al., 2015).

Para os lactentes que tinham anquiloglossia anterior, a maioria (78\%) obteve algum grau de melhora imediata na amamentação após a intervenção, com a maioria (61\%) relatando uma melhora significativa. Aqueles com anquiloglossia posterior, (91\%) relataram algum grau de melhora imediata após a cirurgia, com a maioria (55\%) referindo melhora moderada na amamentação. Resultados favoráveis semelhantes foram observados para participantes com ligamento do lábio superior (100\% melhorado), anquiloglossia anterior e ligamento do lábio superior (91\% melhorado) e anquiloglossia posterior e ligamento do lábio superior (85\% melhorado) (Pransky, et al., 2015). É possível verificar na Tabela 3, as características principais extraídas e descritas de cada artigo admitido nesta pesquisa. 
Tabela 3. Síntese dos artigos incluídos.

\begin{tabular}{|c|c|c|c|c|c|}
\hline $\begin{array}{l}\text { Autor/ Ano/ } \\
\text { Local de } \\
\text { publicação }\end{array}$ & Objetivo & n & Exames & Resultados & Conclusão \\
\hline $\begin{array}{c}\text { Ngernchamet } \\
\text { al., } \\
2013 \\
\text { Tailândia }\end{array}$ & $\begin{array}{l}\text { Prevalência recém- } \\
\text { nascidos com frênulo } \\
\text { lingual e possíveis } \\
\text { dificuldades } \\
\text { subsequentes de } \\
\text { amamentação, assim } \\
\text { como outros fatores } \\
\text { que afetam a eficácia } \\
\text { da amamentação }\end{array}$ & $\begin{array}{l}2.679 \text { mães } \\
\text { e seus bebês }\end{array}$ & $\begin{array}{l}\text { Exame } \\
\text { físico nos } \\
\text { bebês e nas } \\
\text { mães }\end{array}$ & $\begin{array}{l}\text { Cerca de } 247 \text { bebês }(9,2 \%) \\
\text { foram diagnosticados com } \\
\text { dificuldades de amamentação. } \\
\text { As taxas de dificuldade para } \\
\text { amamentar em bebês com } \\
\text { frênulo lingual moderado e } \\
\text { grave foram de } 11,8 \%(71 / 600 \\
\text { bebês) e } 37,9 \%(162 / 428 \\
\text { bebês), respectivamente. }\end{array}$ & $\begin{array}{l}\text { Bebês com frênulo } \\
\text { lingual encurtado não é } \\
\text { incomum e está } \\
\text { associado à dificuldade } \\
\text { em realizar a } \\
\text { amamentação em } \\
\text { recém-nascidos. }\end{array}$ \\
\hline $\begin{array}{l}\text { Pransky et } \\
\text { al., } \\
2015 \\
\text { Estados } \\
\text { Unidos }\end{array}$ & $\begin{array}{c}\text { Descrever as } \\
\text { dificuldade de } \\
\text { amamentação com } \\
\text { foco na anquiloglossia } \\
\text { posterior e laços do } \\
\text { lábio superior. }\end{array}$ & 618 & $\begin{array}{l}\text { Exame } \\
\text { completo de } \\
\text { cabeça e } \\
\text { pescoço nos } \\
\text { lactentes }\end{array}$ & $\begin{array}{c}\text { Cerca de }(\mathrm{n}=290,47 \%) \text { bebês } \\
\text { apresentavam anquiloglossia } \\
\text { anterior. Houve }(\mathrm{n}=120,19 \%) \\
\text { lactentes que foram } \\
\text { considerados como tendo } \\
\text { anquiloglossia posterior e } \\
(\mathrm{n}=14,2 \%) \text { foram encontrados } \\
\text { com ligamento do lábio } \\
\text { superior. Trinta e quatro }(6 \%) \\
\text { lactentes apresentavam } \\
\text { anquiloglossia anterior e } \\
\text { ligamento no lábio superior, } \\
\text { enquanto (n=33, 5\%) } \\
\text { apresentavam anquiloglossia } \\
\text { posterior e ligamento no lábio } \\
\text { superior. }\end{array}$ & $\begin{array}{l}\text { Anquiloglossia anterior e } \\
\text { posterior e ligamento do } \\
\text { lábio superior, ou } \\
\text { combinações dos } \\
\text { mesmos, foram } \\
\text { comumente } \\
\text { reconhecidos em nossa } \\
\text { população de estudo. } \\
\text { Muitos recém-nascidos, } \\
\text { entretanto, também não } \\
\text { apresentavam anomalias } \\
\text { da cavidade oral. } \\
\text { Embora a causa não } \\
\text { possa ser implícita, essas } \\
\text { anomalias da cavidade } \\
\text { oral podem contribuir } \\
\text { para as dificuldades de } \\
\text { amamentação em alguns } \\
\text { casos. }\end{array}$ \\
\hline
\end{tabular}

Fonte: Ngerncham, Laohapensang, Wongvisutdhi et al., (2013); Pransky, Lago, Hong (2015).

\section{Discussão}

Segundo o CID-10 (Classificação Internacional de Doenças e Problemas Relacionados à Saúde), a anquiloglossia é classificada como uma malformação congênita da língua (OMS, 2008). Ela pode ser justificada pela ausência de apoptose na face inferior da língua no período embrionário, podendo ser classificada como anquiloglossia total ou parcial e, estas variações, podem resultar na diminuição dos movimentos linguais, como protrusão, retração, lateralização e elevação (Martinelli et al., 2013; Marcione et al., 2016).

O primeiro estudo (Ngerncham, et al.,2013) relata a presença de alteração do frênulo lingual grave em $16 \%$ dos pacientes e moderada em 22,4\%, o que está condizente com a literatura encontrada, que refere uma prevalência de anquiloglossia variando de 2,2\% a 22\% (Schimitt, et al.,2012; Martinelli, et al., 2013; Simão, 2016; Marcione, et al., 2016). No mesmo estudo autores ainda referem a presença de dificuldade de amamentação em 9,2\% dos pacientes estudados com alteração do frênulo lingual. No segundo estudo (Pransky, et al., 2015), o índice de anquiloglossia foi de 19\%, estando dentro dos padrões encontrados na literatura, porém também relatam a ocorrência de anquiloglossia anterior em $47 \%$ sendo maior do que o esperado.

Em relação à prevalência considerando o sexo, os autores do primeiro estudo (Ngerncham, et al.,2013) referiram que a prevalência do frênulo lingual alterado foi um pouco maior em meninas do que em meninos. Este achado vai de encontro a outro estudo o qual salientou que o frênulo lingual alterado é mais frequente em meninos do que em meninas (Marcione, et al., 
2016). Outras pesquisas salientam que não há diferença entre o sexo feminino e masculino ao se considerar a anquiloglossia (Braga, et al., 2009). Dessa forma, pode-se concluir que não há achados suficientes para afirmar que a alteração do frênulo lingual seja mais comum em meninos ou em meninas. No segundo estudo os autores não mencionaram esta relação.

Já sobre a interferência do frênulo lingual alterado nas funções do sistema sensório motor oral, no primeiro estudo Ngerncham, et al. (2013) referiu que 9,2\% dos bebês com frênulo lingual alterado apresentaram dificuldades de amamentação, sendo a alteração presente considerada de moderada a grave. Estudos evidenciam que, na presença da alteração associada às dificuldades na amamentação, duas categorias de sinais e sintomas podem surgir: os relacionados com o trauma mamilar e os relacionados com a amamentação ineficaz e consequente baixa ingestão nutricional da criança. Ainda, Campanha et al. (2019) relatam que houve relação entre queixa de dificuldade de amamentação com a alteração do frênulo. Dessa forma, concluíram que o diagnóstico da anquiloglossia é importante e deve ser realizado, de forma precoce, ainda nas maternidades.

Pransky, Lago, Hong (2015), no segundo estudo, afirmam que muitos dos recém-nascidos que apresentaram dificuldades na amamentação, 27\%, não apresentavam anomalias na cavidade oral. Segundo a Organização Mundial da Saúde (OMS, 2020), a amamentação exclusiva deve ser realizada até os seis meses, pois traz muitos benefícios para o bebê, principalmente a proteção contra infecções gastrointestinais. Além disso, diversos autores abordam a importância da amamentação para o desenvolvimento das estruturas do sistema estomatognático, auxiliando a mastigação adequada e, consequentemente, a fala precisa (Tanigute, 2005; Silva et al., 2009; Martinelli et al., 2013).

$\mathrm{O}$ recém-nascido com frênulo alterado pode ter sua amamentação prejudicada, o que facilita o desmame precoce, afetando a nutrição e o desenvolvimento do sistema estomatognático. Pompéia et al. (2017), não faz uma relação clara sobre o impacto da anquiloglossia na sucção nutritiva do seio materno, entretanto aponta para relação acerca da influência de frênulo lingual curto, sobre o crescimento e desenvolvimento do sistema estomatognático, bem como sobre a forma-função.

Alterações do sistema estomatognático levam a dificuldades na mastigação, o que retroalimenta as alterações musculares e consequentemente o crescimento ósseo, além de poder impactar na fala, mais especificamente na articulação de fones que necessitam de maior refinamento de movimento e precisão de língua. Mills et al. (2019) salientam que em alguns sujeitos, a variação anatômica da morfologia do frênulo pode criar limitação no movimento da língua, levando a um desequilíbrio entre estabilidade e mobilidade. Pesquisas relacionadas à biomecânica da língua em tarefas específicas nos ajudam a compreender como a restrição de movimento causada pelo frênulo lingual pode impactar variavelmente nas diferentes funções exercidas por ela, sendo a fala uma delas (Geddes, et al. 2008; Xu, 2016).

\section{Considerações Finais}

As pesquisas que abordam a interferência do frênulo lingual nas funções orais, em sua maioria, se relacionam com a sucção e a amamentação, porém ainda são em pequeno número. Quando se trata das funções de mastigação, deglutição e fala, os estudos são ainda mais escassos. No caso do presente estudo nenhum dos artigos apresentaram esta relação. Anquiloglossia anterior e posterior e ligamento do lábio superior, ou combinações dos mesmos, foram comumente reconhecidos nos estudos da revisão sistemática. Embora a causa não possa ser implícita, essas anomalias da cavidade oral podem vir a contribuir para as dificuldades de amamentação em alguns casos.

Com base nos achados, percebe-se que bebês com frênulo lingual alterado não são incomuns e esta alteração pode estar associada à dificuldade em realizar a amamentação em recém-nascidos, porém como mencionado, pela escassez de estudos voltados para as outras funções, não se pode afirmar que a alteração do frênulo tenha relação com a alteração de todas as funções do sistema sensório motor oral, como mastigação, deglutição e fala. Sugere-se para próximas pesquisas ampliar o número de participantes nos estudos, visando detalhar e especificar de forma mais concisa os dados para a avaliação. 
Research, Society and Development, v. 10, n.1, e3510111396, 2021 (CC BY 4.0) | ISSN 2525-3409 | DOI: http://dx.doi.org/10.33448/rsd-v10i1.11396

\section{Referências}

Bervian, J., Fontana. M., \& Caus, B. Relação entre amamentação, desenvolvimento motor bucal e hábitos bucais - revisão de literatura. Revista da Faculdade de Odontologia - UPF, 13(2).

Braga, L. A. S., Silva, J., Pantuzzo, C. L., \& Motta, A. R. Prevalência de alteração no frênulo lingual e suas implicações na fala de escolares. CEFAC. 2009. 11 (Suppl 3): 378-390.

Brito, S. F., Marchesan, I. Q., Bosco, C. M., Carrilho, A. C. A., \& Rehder, M. I. Frênulo lingual: classificação e conduta segundo ótica fonoaudiológica, odontológica e otorrinolaringológica. CEFAC. 2008; 10 (3): 343-351.

Campanha, S. M. A., Martinelli, R. L. C, \& Palhares, D. B. Association between ankyloglossia and breastfeeding. CODAS. 2019;31(1):e20170264.

Campos, B., Susanibar, F., Carranza, C. A., \& Oliveira, N. C. M. (2015). Embriologia do sistema estomatognático. In: Susanibar, F., Marchesan, I. Q., Ferreira, V. J. A., Douglas, C. R., Parra, D., Dioses, A. Motricidade Orofacial: Fundamentos Neuroanatômicos, Fisiológicos e Linguísticos. Ribeirão Preto: Book Toy. 23-60.

Fraga, M. R. B. A., Lira, T. C. B., Celerino, P. R. R. P., Tavares, I. T. S., \& Menezes, V. A. Anquiloglossia versus amamentação: qual a evidência de associação? CEFAC. 22(3): e12219.

Geddes, D., Langton, D., Gollow, I., Jacobs, L., Hartmann, P., \& Simmer, K. Frenulotomy for breastfeeding infants with ankyloglossia: effect on milk removal and sucking mechanism as imaged by ultrasound. Pediatrics. 2008;122(1):e188-94.

Gonçalves, C. S., \& Ferreiro, M. C. Estudo da relação entre presença de frênulo lingual curto e/ou anteriorizado e a dorsalização de [r] na articulação da fala. CEFAC. 8(1): 56-60.

Manipon, C. Ankyloglossia and the breastfeeding infant: assessment and intervention. Adv Neonatal Care. 2016;16(2):108-13.

Marchesan, I. Q., Martinelli, R. L. C., \& Gusmão, R. J. Frênulo lingual: modificações após frenectomia. Rev Soc Bras Fonoaudiol. $2012 ; 24$ (4): $409-412$.

Marcione, E. S. S. Classificação anatômica do frênulo lingual de bebês. CEFAC. 2016; 18 (5): 1042-1049.

Martinelli, R. L. C., Marchesan, I. Q., \& Berretin-Felix, G. Protocolo de avaliação do frênulo lingual para bebês: relação entre aspectos anatômicos e funcionais. Revista Cefac.2013; 15(3): 599-610.

Mills, N., Pransky, S. M., Geddes, D. T., \& Mirjalili, S. A. What is a tongue tie? Defining the anatomy of the in-situ lingual frenulum. Clin Anat. 2019.

Moher, D., Shamseer, L., \& Clarke, M., et al. Preferred reporting items for systematic review and meta-analysis protocols (PRISMA-P) 2015 statement. Syst Rev. 4(1): 1

Ngerncham, S., Laohapensang, M., Wongvisutdhi, T., Ritjaroen, Y., Painpichan, N., \& Hakularb, P., et al. Lingual frenulum and effect on breastfeeding in Thai newborn infants.Paediatr Int Child Health. 33(2), 86-90.

Organização Mundial De Saúde. Classificação Estatística Internacional De Doenças E Problemas Relacionados À Saúde - CID-10. [publicação online]; 2008.

Pithon, M. M., Sant'anna, L. I. D. A., Baião, F. C. S., Santos, R. L., Coqueiro, R. S, \& Maia, L. C. (2015). Assessment of the effectiveness of mouthwashes in reducing cariogenic biofilm in orthodontic patients: a systematic review. 43:297-308.

Pompeia, L. E. A influência da anquiloglossia no crescimento e desenvolvimento do sistema estomatognático. São Paulo: Paul Pediatr, 2017.

Pransky, S. M., Lago, D., \& Hong, P. (2015). Breastfeeding difficulties and oral cavity anomalies: the influence of posterior ankyloglossia and upper-lip ties.Int. J. Pediatr. Otorhinolaryngol. 79(10), 1714-7.

Rios, I. J. A. Estimulação da Sucção para Recém-Nascidos de Alto Risco. In: Marchesan IQ. Fundamentos em Fonoaudiologia: aspectos clínicos da motricidade oral. (2a ed.), Rio de Janeiro: Guanabara, 2005. Cap. 1, 1-9.

Silva, M. C, et al. (2009), Frênulo de língua alterado e interferência na mastigação. Rev. CEFAC. 11 (3): 363-369.

Schmitt, B. H. E., Guzzi, S. H., Damo, M. N., Araújo, S. M., \& Farias, M. M. A. G. (2012). Características da cavidade oral de bebês recém-nascidos, Blumenau/SC. Pesqui. bras. odontopediatria clín. integr., 12(1).

Simão, C. A. B. (2016). Prevalência De Anquiloglossia Em Recém-Nascidos E Avaliação Da Confiabilidade E Validade Do Teste Da Linguinha Na Triagem Neonatal. 100 f. Dissertação (Mestrado em Odontologia) - Faculdade de Odontologia, Universidade do Estado do Rio de Janeiro.

Tanigute, C. C. Desenvolvimento das Funções Estomatognáticas. Irene Queiroz. Fundamentos em Fonoaudiologia: aspectos clínicos da motricidade oral. (2a ed.), Rio de Janeiro: Guanabara, 2005. Cap. 1, 1-9.

World Health Organization. (2020). Infant and Young child nutrition. Fifty-fourth World Health Assembly. Geneva: World Health Assembly (WHA).

Xu, K. (2016).3D tongue motion visualization based on the B-mode ultrasound tongue images [tesis]. Paris: Computer Aided Engineering, Université Pierre et Marie Curie. 